\title{
Aberrant structural and functional connectivity in the salience network and central executive network circuit in schizophrenia
}

\author{
Quan Chen ${ }^{\mathrm{a}}$, Xiaoxuan $\mathrm{He}^{\mathrm{a}}$, Xingui Chen ${ }^{\mathrm{b}}$, Lu Wang ${ }^{\mathrm{a}}$, Kai Wang ${ }^{\mathrm{b},{ }^{*}}$, Bensheng Qiu $^{\mathrm{a}, \mathrm{c}^{* * *}}$
}

${ }^{a}$ Center for Biomedical Engineering, University of Science and Technology of China, Hefei, Anhui 230027, China

${ }^{b}$ Department of Neurology, The First Affiliated Hospital of Anhui Medical University, Hefei, Anhui 230022, China

${ }^{c}$ Anhui Computer Application Institute of Traditional Chinese Medicine, Hefei, Anhui 230038, China

\footnotetext{
Abbreviations: FA, fractional anisotropy; SN, salience network; CEN, central executive network; IFOF, inferior fronto-occipital fasciculus; ILF, inferior long fasciculus; SFOF, superior fronto-occipital fasciculus; SLF, superior long fasciculus; UF, uncinate fasciculus. DLPFC, dorsolateral prefrontal cortex; PPC; posterior parietal cortex; ACC, anterior cingulate cortex; FIC; fronto-insular cortex.

*Corresponding author at: Department of Neurology, the First Affiliated Hospital of Anhui Medical University, Hefei, Anhui Province 230022, PR China.

**Corresponding author at: Center for Biomedical Engineering, University of Science and Technology of China, Hefei, Anhui 230027, PR China.

E-mail address: wangkai1964@126.com (K.Wang) and bqiu@ustc.edu.cn (Bensheng Qiu)
} 


\section{Abstract}

Consistent structural and functional abnormities have been detected in the salience network $(\mathrm{SN})$ and the central-executive network (CEN) in schizophrenia. SN, known for its critical role in switching CEN and default-mode network (DMN) during cognitively demanding tasks, is proved to show aberrant regulation on the interaction between DMN and CEN in schizophrenia. However, it has not been elucidated whether there is a direct alteration of structural and functional connectivity between SN and CEN. 22 schizophrenia patients and 21 healthy controls were recruited for functional magnetic resonance imaging (fMRI) and diffusion tensor imaging (DTI) in present study. The results show that schizophrenia patients had lower fractional anisotropy (FA) in right inferior long fasciculus (ILF), left inferior fronto-occipital fasciculus (IFOF) and callosal body than healthy controls. Significantly reduced functional connectivity was also found between right fronto-insular cortex (rFIC) and right posterior parietal cortex (rPPC). FA in right ILF was positively correlated with the functional connectivity of rFIC-rPPC. Therefore, we proposed a disruption of structural and functional connectivity and a positive anatomo-functional relationship in SN-CEN circuit, which might account for a core feature of schizophrenia.

Keywords: schizophrenia, fractional anisotropy (FA), functional connectivity, salience network (SN), central executive network (CEN)

\section{Introduction}

Schizophrenia is a complex mental disorder characterized by a multitude of symptoms affecting a variety of emotional and cognitive domains $[1,2]$. The symptoms themselves have been linked to the malfunction of multiple large-scale distributed intrinsic connectivity networks [3]. In particular, two canonical networks have received increasing attention for their potential relevance to schizophrenia: salience network (SN) and central executive network (CEN)[4, 5]. The $\mathrm{SN}$, anchored on insula and the anterior cingulate cortex (ACC), plays an important role in detecting and processing of cognitive, emotional and homeostatic salience events[6]. The CEN, whose key nodes include the dorsolateral prefrontal cortex (DLPFC) and posterior parietal cortex 
(PPC), is usually observed to be active in task-positive tasks due to their roles in attention, working memory and response selection [7]. Dysfunction within the two brain networks have been demonstrated in schizophrenia $[1,5,8-10]$. It has been reported that positive symptoms like hallucinations or passivity experiences may result from abnormal salience to inner speech or other self-generated actions[5, 11]. Meanwhile, CEN may be linked to dysfunctional outcomes in schizophrenia such as impairment of working memory, attention, inhibition and task management $[1,8-10]$.

Earlier investigations suggested the coactivation of SN and CEN in cognitively demanding tasks[12], while evidences for their flexible, dynamic relationship started to be reported recently $[3,7,13]$. Sridharan et.al explored the temporal relationship of SN activity relative to CEN and DMN activity. They found that onset latency is consistently and significantly lower in SN when compared to regions of the CEN and DMN. Furthermore, they observed that right FIC activity Granger-causes ACC activity and has a powerful causal influence on the other regions. The result revealed an important role of SN in switching the two networks [7]. Besides, a decreased time-lagged connectivity between $\mathrm{SN}$ and CEN was observed in the ongoing blood-oxygenation-level dependent activity in schizophrenia patients, suggesting that the abnormal connectivity between SN and CEN may exert an influence on schizophrenia [3].

Based on these findings, the question arises as what is the underlying basis for the abnormal effective causal SN-CEN interaction. Recently, few studies started to focus on the white matter tracts connecting the SN-CEN circuit [14], which showed that inferior fronto-occipital fasciculus (IFOF), superior long fasciculus (SLF), inferior long fasciculus (ILF) and uncinate fasciculus (UF) account for the anatomic connectivity in SN-CEN circuit. The ILF may be of great relevance, as it connects the parietal and occipital lobes with anterior temporal cortex and joins the UF to connect the frontal lobes, comprising the SN-CEN circuits directly or indirectly[15]. In schizophrenia, abnormalities of ILF led to neurocognitive deficits and visual hallucinations[16, 17]. It is deducible that alteration in the tracts within SN-CEN may have an influence on functional dysconnectivity between the two networks, and further be the potential cause for aberrant temporal causal SN-CEN/DMN interaction. Therefore, by combining fMRI and DTI to assess the connectivity alterations in SN-CEN circuit, the present study seeks to address whether 
schizophrenia patients show significant reduction in structural and functional connectivity in SN-CEN circuit, and whether impaired integrity of white matter tracts correlates with changed function connectivity in schizophrenia.

\section{Methods}

\subsection{Participants}

Twenty-two schizophrenia patients and twenty-one healthy controls without significant difference in age, sex and years of education were recruited for this study. Signed informed consent in accordance with ethical committee of Anhui Medical University were obtained before the study. The structured clinical interview of DSM-IV (SCID) was used to diagnose schizophrenia, and the severity of clinical symptom was assessed by positive and negative syndrome scale (PANSS). The DSM-IV non-patient edition (SCID-NP) was used to evaluate healthy participants (Table.1). All patients were outpatients and received antipsychotic medication, including Risperidone $(\mathrm{n}=6)$, Chlorpromazine $(\mathrm{n}=3)$, Clozapine $(\mathrm{n}=2)$, Aripiprazole $(\mathrm{n}=1)$, Aripiprazole + Clozapine $(n=4)$, Aripiprazole + Risperidone $(n=2)$, Aripiprazole + Quetiapine $(\mathrm{n}=2)$, Quetiapine + Risperidone $(\mathrm{n}=1)$ and Risperidone + Clozapine $(\mathrm{n}=1)$. Risperidone equivalent doses (Supplementary Table.S1 )were computed for oral antipsychotic medication using method presented by Nancy C. Andreasen [18]. All controls were free of any psychiatric disorder and psychotropic medication. Individuals with any of the following situations were excluded: pregnancy, other psychiatric symptoms, substance abuse, MRI contraindications and any other condition that might affect the experiment.

\subsection{MRI data acquisition}

All MRI scanning were conducted on a GE 3T MRI scanner (GE Medical System, Milwaukee, Wisconsin) equipped with a 8-channel head coil. T1-weighted anatomic images were acquired in sagittal orientation with three-dimensional inversion recovery prepared fast spoiled gradient recalled sequence of the following parameters: repetition time/echo time ratio $=8.676 / 3.184 \mathrm{~ms}$, 
inversion time $=800 \mathrm{~ms}$, flip angle $=8$ degrees, field of view $=256 \times 256 \mathrm{~mm}^{2}$, matrix size $=256$ $\times 256$, slice thickness $=1 \mathrm{~mm}$ and the number of slices is 188 . The diffusion weighted data were acquired using echo planar imaging sequence with $\mathrm{TR} / \mathrm{TE} / \mathrm{FA}=1100 \mathrm{~ms} / 72 \mathrm{~ms} / 90^{\circ}$, number of slices $=50$, slice thickness $=3 \mathrm{~mm}$, matrix $=256 \times 256$. A total of 64 independent directions with a diffusion-weighted factor of $b=1000 \mathrm{~s} / \mathrm{mm}^{2}$ were applied and three B0 images were collected at the beginning of the process. For the resting state fMRI data, 240 slices were scanned in 8 min with an EPI_BOLD sequence $\left(33\right.$ slices, $T R=2000 \mathrm{~ms}, \mathrm{TE}=22.5 \mathrm{~ms}, \mathrm{FA}=90^{\circ}$, matrix $=64 \times 64$, field of view $=220 \times 220 \mathrm{~mm}^{2}$, slice thickness $\left.=4 \mathrm{~mm}\right)$. The first 4 scans were used to achieve equilibrium and were not included in the analysis. All participants were instructed to keep their eyes closed and avoid falling asleep or getting distracted during the scan. A T2-wighed sequence was scanned to diagnose obvious brain disease.

\subsection{Data preprocessing and analysis}

\subsubsection{DTI data analysis}

The preprocessing of DTI data and tract-based spatial statistics (TBSS) analysis were done using FSL (FMRIB Software Library, http://fsl.fmrib.ox.ac.uk/fsl/fslwiki/TBSS). After correction for the effects of head motion and eddy currents and registration to a Montreal Neurological Institute (MNI152) common space, the map of FA for each person was generated. Then, all aligned FA images was thinned to generate a mean FA skeleton, which represented the center of all tracts common to the group. A threshold of 0.2 of the mean FA skeleton was set to confine the analysis to white matter. The aligned FA data of each subject were subsequently projected onto this skeleton by searching for the local center of the nearest relevant fiber tract. Thus, for each subject an individual FA skeleton was produced.

A voxel-wise statistical analysis was performed to detect significant difference in FA between patients and healthy controls by using the FSL Randomize program. Both $\mathrm{HC}>\mathrm{SC}$ and $\mathrm{SC}>\mathrm{HC}$ contrasts were tested with 5000 permutations and the significance level was set at $p<0.05$. The results were corrected for multiple comparisons using the threshold-free cluster enhancement (TFCE) method with family wise-error (FWE). The JHU White-Matter Tractography Atlas in FSL 
toolbox was used to identify the abnormal WM fiber tracts.

Atlas-based segmentation method was used to confirm the result of TBSS. Region-of-interest masks (ILF (left/right), UF (left/right), SLF (left/right), IFOF (left/right), superior fronto-occipital fasciculus (SFOF, left/right) and Callosal body) were extracted from the JHU White-Matter Tractography Atlas and Juelich White-Matter Tractography Atlas provided by FSL .The probability threshold was set at 25 . Mean diffusion parameters (FA, radial diffusivity (RD), and mean diffusivity (MD)) in each mask were calculated by a customized MATLAB script (MATLAB, The MathWorks). Intergroup comparisons were then performed using two-sample unpaired t-tests $(\mathrm{p}<0.05)$, Bonferroni-corrected for multiple comparisons. Age, sex and education were controlled as covariates.

\subsection{2 fMRI data analysis}

Resting-state fMRI data was analyzed with joint utilization of AFNI (Medical College of Wisconsin, Milwaukee, Wisconsin, USA) and FSL programs. Pre-processing steps include slice timing correction, realignment, skull stripping, coregisteration and normalization into the MNI152 standard brain, band-pass filtering $(0.01-0.08 \mathrm{~Hz})$ and spatial smoothing using a 6-mm full width at half maximum Gaussian kernel. Data with head motion amplitude over $2 \mathrm{~mm}$ was excluded. To minimize the effect of head motion, relative head motion was generated by calculating the temporal differential of the head motion. Volume with relative motion over $0.2 \mathrm{~mm}$ was censored out (More details in Supplementary Table.S2).

Functional connectivity was calculated as follow. Firstly, according to the research [7], we defined 8 regions of interest (ROIs) in 4 canonical nodes to represent CEN and SN. ROI masks (DLPFC (left/right), PPC (left/right), ACC (left/right), FIC (left/right)) were created using the predefined TD brodmann areas atlas in WFU Pickatlas (Table.2) and mean time course of each ROI was extracted. Then, the Pearson's correlation coefficients for all pair-wise time-courses were calculated, followed by Z-Fisher's r-to-z transformation to improve normality. An $8 \times 8$ correlation matrix was produced for each subject. Finally, two-sample unpaired t-test was used to compare the functional connectivity between the groups ( $\mathrm{p}<0.05$, Bonferroni-corrected, controlled for age, sex 
and education). BrainNet Viewer were applied for visualization.

\subsubsection{Correlation analysis}

Pearson correlative analysis $(\mathrm{p}<0.05)$ was used to investigate the correlation between altered FA in right ILF and the aberrant functional connectivity of rFIC-rPPC in two groups. Age, sex and education were set as covariates. Fisher z-transformation was used to investigate the difference between two correlation coefficients.

\section{Results}

3.1 The structural connectivity (DTI results) in SN-CEN circuit

FA values in white matter tracts were measured by TBSS and atlas-based segmentation method. Both TBSS (FIG.1; Supplementary FIG.S1, FIG.S2) and atlas-based segmentation (Table.3) showed FA reduction in white matter tracts in schizophrenia patients. No significant difference in MD and RD were detected (Supplementary Table.S3, Table.S4).

\subsection{The functional connectivity (fMRI results) in SN-CEN circuit}

The main effect analysis results revealed a significantly reduced functional connectivity between $\mathrm{rFIC}$ and $\mathrm{rPPC}$ in schizophrenia patients (Table.4). BrainNet Viewer were used to visualize the functional brain networks (FIG.2).

3.3 Relationship between the structural connectivity and functional connectivity in SN-CEN circuit

Significant correlation had been detected in patients between FA in ILF-R and functional connectivity of rFIC-rPPC. (Table5, FIG.3). No significant anatomo-functional correlation could be found in healthy controls. The p-value of Fisher z-transformation is 0.0497 . 


\section{Discussion}

Reduced FA was detected in several white matters, especially in ILF, IFOF and Callosal body, which connect the SN-CEN circuit. Meanwhile, decreased functional connectivity was observed within the circuit: rFIC-rPPC. Furthermore, FA in right ILF was positively correlated with the functional connectivity of rFIC-rPPC. Therefore, the present study suggested an anatomo-functional dysconnectivity within SN-CEN circuit in schizophrenia.

\subsection{Diffusion abnormalities}

Callosal body facilitates interhemispheric communication. IFOF and ILF are reported to connect SN and CEN [14]. These tracts are altered in schizophrenia [19], which is consistent with our study. Decreased FA has been detected in parts of the corpus interconnecting frontal regions[20]. Integrity of callosal fibers is correlated with positive and negative symptoms[20]. IFOF connects the visual system with the frontal cortex and is thought to be a key pathway in processing information in sensory-SN-CEN loop. In schizophrenia patients, this hierarchical process has been disrupted by reduced visual inflow to and frontal outflow from the anterior insula [21], which is in line with the finding of reduced FA in the white matter tract [22].

The ILF is composed of two parts: a vertical limb in the parietal and occipital lobe that connects the gray matter in Brodmann areas 7 and 39 in parietal cortex and Brodmann areas 17,18,19,37 and 39 in occipital lobe; a horizontal component contained within the temporal lobe with axonal projections from Brodmann areas 20,21,22 and 38[15, 23]. It is an important source of fibers afferent to amygdala [24], which is also an important node of salience network[25]. Moreover, the anterior temporal part of ILF is in the skirt of insula cortex[19], and it joins the UF to connect brain areas like Brodmann areas 47,13 and 14 in an indirect pathway and relays information to the orbito-frontal brain[15, 23]. Besides, along part of the pathway, ILF spatially overlaps with the IFOF, which connects the frontal cortex with the occipital lobe and plays an important role in SN-CEN circuit[26]. Thus, the ILF can provide an anatomic pathway for SN-CEN circuit in a direct way or in an indirect way. Schizophrenia patients have significantly 
decreased FA and increased RD in this region. And the decreased white matter integrity is related to visual hallucinations[16]. Combining the previous studies with our findings, we propose a disruption of the structural connectivity within SN-CEN circuit in schizophrenia, of which impaired integrity of ILF may exert a powerful influence.

\subsection{Functional connectivity abnormalities}

Our study demonstrated functional abnormalities in patients with schizophrenia compared with healthy participants. Patients showed decreased functional connectivity between FIC and PPC. This result is consistent with a previous study, which showed that the temporal causal connectivity between SN and dorsal CEN is disrupted in schizophrenia [3]. In present study, we chose FIC/ACC and DLPFC/PPC to represent SN and CEN, respectively. The 4 regions are reported to be the key nodes or hubs of corresponding networks $[4,7,14,27]$. When investigating the relationship between SN and CEN/DMN, Sridharan et.al found that only FIC and ACC of the SN consistently show peak latency differences with the CEN and DMN regions across different tasks. Then they included FIC and ACC, along with DLPFC and PPC of CEN for network analysis [7]. Also, Andrei Manoliu suggested the time-lagged connectivity between SN and CEN is reduced in schizophrenia while the direct functional connectivity change between the networks is not apparent after multiply comparisons correction [3]. Meanwhile, mounting evidences indicated that FIC, ACC, DLPFC and PPC play a critical role in cognitive and emotional processes, with their aberrant activation possibly related to the worse neurological performance in schizophrenia patients $[5,28,29]$. For the exploration purpose, we chose bilateral regions of FIC, ACC, DLPFC and PPC to investigate the functional connectivity between SN and CEN.

\subsection{Potential mechanism for anatomo-functional dysconnectivity}

Apart from alterations in the structural and functional connectivity in the SN-CEN circuit, a significant positive correlation was found between FA in right ILF and functional connectivity of rFIC-rPPC in patients. The reduced FA may reflect an alteration in axonal diameter, fiber organization or fiber density and represent fiber demyelination through disturbances in glial cell 
morphology [30, 31]. It is deducible that a decrease in the quantity of axon coupled with demyelination in ILF may result in the functional dysconnectivity of SN-CEN circuit. And the possible mechanism may be a slower and less efficient signal transfer through myelin sheaths and changed axon [32], which can be supported by a previous study in mice showing that reduction of propagation velocity in white matter tracts is related to the loss of myelination [33]. Whereas, there was no significant correlation between functional and structural connectivity in the healthy controls, which was consistent with previous studies[32]. Such absence may result from the high value of functional connectivity ( $\mathrm{Z}$ value) and FA value and the low variation of these values, as healthy participants have comparative intact brain structure when compared with schizophrenia patients.

\subsection{Positive anatomo-functional relationship in SN-CEN}

In schizophrenia, a direct positive correlation between changed anatomic connectivity and altered functional connectivity has been explored recently in different tracts and networks, but not in SN-CEN circuit. Using the TBSS approach in the whole brain, Jeong et al. showed that the decreased mean FA value in the left inferior frontal white matter regions is positively related to the functional connectivity abnormalities between left inferior frontal gyrus and left middle temporal gyrus during semantic encoding [19]. Gerd Wagner et al. reported that decreased fronto-thalamic effective connectivity is attributed to lower FA in the right anterior limb of the internal capsule (ALIC) [30]. Overall, these multimodal studies have revealed that impaired integrity of white matter can transform into decreased functional connectivity in schizophrenia. Most studies investigating the association among regions in SN-CEN circuit are based on temporal lagging relationship between the two networks, while the direct functional connectivity change among the networks is not apparent [3]. Others have investigated the structural and causal effective connectivity in SN-CEN circuit, with no positive anatomo-functional relationship found in schizophrenia patients [14]. Thus, present study provides the first indication that an impaired structural connectivity in schizophrenia within the ILF is accompanied with a reduction in functional connectivity of SN-CEN circuit in schizophrenia. 


\section{Limitations and Further points}

In current study, patients were recruited with antipsychotic treatment. However, we have reason to believe that the alterations of connectivity is due to the disease, as the patients' PNASS scores are higher than 10. A more rigorous research design will be need, such as including first-diagnosed, drug-naive patients in the study. Meanwhile, as most multimodal studies remain limited, further research using tract tracing between regions have to be considered to provide a more accurate evaluation of white matter.

\section{Conclusion}

The present study reveals deficits in structural and functional connectivity and a positive relationship of anatomo-functional disruption within SN-CEN circuit in schizophrenia patients. The finding suggests that the abnormal white matter integrity in tracts associated with the SN-CEN circuit might be the main reason for functional dysconnectivity in the circuit and consequently the failing modulation of $\mathrm{SN}$ to switch CEN/DMN. The altered FA in white matter tracts, associated with reduced functional connectivity in schizophrenia patients strongly supports the hypothesis of a disconnection in SN-CEN circuit.

\section{Contributors}

Q. Chen participated in experimental design, data processing and paper preparation. X. $\mathrm{He}$ and L. Wang participated in data processing and paper revision. X. Chen participated in data acquisition and paper revision. K. Wang and B. Qiu participated in experimental design and paper revision.

\section{Acknowledgements}

This work was supported by National Science Foundation of China (Grant number: 81371537, 81171273, 31571149, 91432301), and Fundamental Research Funds for the Central 
Universities of China (WK2070000033).

\section{Conflicts of interest}

There are no conflicts of interest.

\section{Reference}

1. Eisenberg, D.P. and K.F. Berman, Executive function, neural circuitry, and genetic mechanisms in schizophrenia. Neuropsychopharmacology, 2010. 35(1): p. 258-77.

2. Gruber, O., A. Chadha Santuccione, and H. Aach, Magnetic resonance imaging in studying schizophrenia, negative symptoms, and the glutamate system. Front Psychiatry, 2014. 5: p. 32.

3. Manoliu, A., et al., Aberrant dependence of default mode/central executive network interactions on anterior insular salience network activity in schizophrenia. Schizophr Bull, 2014. 40(2): p. 428-37.

4. Chang, X., et al., Altered default mode and fronto-parietal network subsystems in patients with schizophrenia and their unaffected siblings. Brain research, 2014. 1562: p. 87-99.

5. Palaniyappan, L. and P.F. Liddle, Does the salience network play a cardinal role in psychosis? An emerging hypothesis of insular dysfunction. Journal of psychiatry \& neuroscience: JPN, 2012. 37(1): p. 17

6. Bressler, S.L. and V. Menon, Large-scale brain networks in cognition: emerging methods and principles. Trends Cogn Sci, 2010. 14(6): p. 277-90.

7. Sridharan, D., D.J. Levitin, and V. Menon, A critical role for the right fronto-insular cortex in switching between central-executive and default-mode networks. Proc Natl Acad Sci U S A, 2008. 105(34): p. 12569-74.

8. Pantelis, C., et al., Spatial working memory deficits in schizophrenia: relationship with tardive dyskinesia and negative symptoms. American Journal of Psychiatry, 2001. 158(8): p. 1276-1285.

9. Stirling, J., et al., Thought disorder in schizophrenia is associated with both executive dysfunction and circumscribed impairments in semantic function. Psychological medicine, 2006. 36(04): p. 475-484.

10. Barch, D.M. and A. Ceaser, Cognition in schizophrenia: core psychological and neural mechanisms. Trends in cognitive sciences, 2012. 16(1): p. 27-34.

11. Kapur, S., Psychosis as a state of aberrant salience: a framework linking biology, phenomenology, and pharmacology in schizophrenia. American journal of Psychiatry, 2003. 160(1): p. 13-23.

12. Fox, M.D., et al., Spontaneous neuronal activity distinguishes human dorsal and ventral attention systems. Proceedings of the National Academy of Sciences, 2006. 103(26): p. 10046-10051.

13. White, T.P., et al., Aberrant salience network (bilateral insula and anterior cingulate cortex) 
connectivity during information processing in schizophrenia. Schizophrenia research, 2010. 123(2): p. 105-115.

14. Iwabuchi, S.J., P.F. Liddle, and L. Palaniyappan, Structural connectivity of the salience-executive loop in schizophrenia. Eur Arch Psychiatry Clin Neurosci, 2015. 265(2): p. 163-6.

15. Schmahmann, J.D., et al., Cerebral white matter. Annals of the New York Academy of Sciences, 2008. 1142(1): p. 266-309.

16. Ashtari, M., et al., Disruption of white matter integrity in the inferior longitudinal fasciculus in adolescents with schizophrenia as revealed by fiber tractography. Archives of general psychiatry, 2007. 64(11): p. 1270-1280.

17. Ellison-Wright, I. and E. Bullmore, Meta-analysis of diffusion tensor imaging studies in schizophrenia. Schizophrenia research, 2009. 108(1): p. 3-10.

18. Andreasen, N.C., et al., Antipsychotic dose equivalents and dose-years: a standardized method for comparing exposure to different drugs. Biological psychiatry, 2010. 67(3): p. 255-262.

19. Jeong, B., et al., Functional and anatomical connectivity abnormalities in left inferior frontal gyrus in schizophrenia. Hum Brain Mapp, 2009. 30(12): p. 4138-51.

20. Kubicki, M., et al., Reduced interhemispheric connectivity in schizophrenia-tractography based segmentation of the corpus callosum. Schizophrenia research, 2008. 106(2): p. 125-131.

21. Palaniyappan, L., et al., Neural primacy of the salience processing system in schizophrenia. Neuron, 2013. 79(4): p. 814-828.

22. Lee, S.-H., et al., Extensive white matter abnormalities in patients with first-episode schizophrenia: a diffusion tensor imaging (DTI) study. Schizophrenia research, 2013. 143(2): p. 231-238.

23. Dick, A.S. and P. Tremblay, Beyond the arcuate fasciculus: Consensus and controversy in the connectional anatomy of language. Brain, 2012: p. aws222.

24. Ortibus, E., et al., Integrity of the inferior longitudinal fasciculus and impaired object recognition in children: a diffusion tensor imaging study. Developmental Medicine \& Child Neurology, 2012. 54(1): p. 38-43.

25. Menon, V. and L.Q. Uddin, Saliency, switching, attention and control: a network model of insula function. Brain Struct Funct, 2010. 214(5-6): p. 655-67.

26. Ashtari, M., Anatomy and functional role of the inferior longitudinal fasciculus: a search that has just begun. Developmental Medicine \& Child Neurology, 2012. 54(1): p. 6-7.

27. Droutman, V., S.J. Read, and A. Bechara, Revisiting the role of the insula in addiction. Trends in cognitive sciences, 2015. 19(7): p. 414-420.

28. Torrey, E.F., Schizophrenia and the inferior parietal lobule. Schizophrenia research, 2007. 97(1): p. 215-225.

29. Zhou, Y., et al., Functional dysconnectivity of the dorsolateral prefrontal cortex in first-episode schizophrenia using resting-state fMRI. Neuroscience letters, 2007. 417(3): p. 297-302.

30. Wagner, G., et al., Structural and functional dysconnectivity of the fronto-thalamic system in schizophrenia: A DCM-DTI study. Cortex, 2015. 66: p. 35-45.

31. Ruest, T., et al., High - resolution diffusion tensor imaging of fixed brain in a mouse model of Pelizaeus -Merzbacher disease: comparison with quantitative measures of white matter pathology. NMR in Biomedicine, 2011. 24(10): p. 1369-1379. 
32. Leroux, E., N. Delcroix, and S. Dollfus, Left fronto-temporal dysconnectivity within the language network in schizophrenia: An fMRI and DTI study. Psychiatry Research: Neuroimaging, 2014. 223(3): p. 261-267.

33. Michailov, G.V., et al., Axonal neuregulin-1 regulates myelin sheath thickness. Science, 2004. 304(5671): p. 700-703. 
Table.1 Participants' demographics

\begin{tabular}{llll}
\hline Characteristics & $\begin{array}{l}\text { HC group } \\
(\mathrm{n}=21)\end{array}$ & $\begin{array}{l}\text { SC group } \\
(\mathrm{n}=22)\end{array}$ & P-value \\
\cline { 2 - 3 } & Mean(SD) & Mean(SD) & \\
\hline Age (years) & $36.10 \pm 7.73$ & $34.05 \pm 7.08$ & 0.369 \\
Gender(F/M) & $11 / 10$ & $17 / 5$ & 0.091 \\
Years of education & $10.05 \pm 2.27$ & $10.27 \pm 2.80$ & 0.774 \\
Illness duration (months) & N/A & $27.09 \pm 2.74$ & N/A \\
PANSS positive & N/A & $15.86 \pm 2.77$ & N/A \\
PANSS negative & N/A & $14.82 \pm 2.02$ & N/A \\
PANSS General & N/A & $57.64 \pm 4.33$ & N/A \\
Risperidone(mg) & N/A & $6.40 \pm 1.34$ & N/A \\
\hline
\end{tabular}

Table. 1 - Characteristics of participants. $\mathrm{p}<0.05$ with $*$ for significant $\mathrm{p}$-value.

Abbreviations: HC, healthy control; SC, schizophrenia patients; F/M, female/male; PANSS, positive and negative syndrome scale; $n$, number of participants; SD, standard deviation; N/A, not applicable. 
Table.2 Coordinates and Abbreviations of SN and CEN regions

\begin{tabular}{llllll}
\hline Region & Abbreviations & BA & \multicolumn{2}{c}{ MNI coordinate } \\
\hline Fronto-insular Cortex (left) & lFIC & 47 & -5 & 17 & 31 \\
Fronto-insular Cortex (right) & rFIC & 47 & 4 & 17 & 31 \\
Anterior Cingulate Cortex (left) & lACC & $24 / 32$ & -25 & 37 & 43 \\
Anterior Cingulate Cortex (right) & rACC & $24 / 32$ & 23 & 37 & 43 \\
Dorsolateral Prefrontal Cortex (left) & lDLPFC & 9 & -36 & 35 & -5 \\
Dorsolateral Prefrontal Cortex (right) & rDLPFC & 9 & 34 & 35 & -5 \\
Posterior Parietal Cortex (left) & IPPC & 40 & -47 & -45 & 45 \\
Posterior Parietal Cortex (right) & rPPC & 40 & 44 & -45 & 44 \\
\hline
\end{tabular}

Table.2 - Coordinates and abbreviations of SN and CEN regions in Brodmann area.

Abbreviations: SN, salience network; CEN, central executive network; BA, Brodmann area. 
Table.3 Mean value of fractional anisotropy (FA) in white matter tracts

\begin{tabular}{|c|c|c|c|c|c|c|c|}
\hline \multirow[t]{2}{*}{ Tracts } & \multirow{2}{*}{$\begin{array}{l}\text { HC group } \\
(\mathrm{n}=21) \\
\text { Mean }(\mathrm{SD})\end{array}$} & \multirow{2}{*}{$\begin{array}{l}\text { SC group } \\
(\mathrm{n}=22) \\
\text { Mean(SD) }\end{array}$} & \multirow[t]{2}{*}{$P$ value } & \multirow[t]{2}{*}{$\begin{array}{l}\text { Degree } \\
\text { of freedom }\end{array}$} & \multirow[t]{2}{*}{$\begin{array}{l}\text { Partial } \\
\text { ETA } \\
\text { square }\end{array}$} & \multicolumn{2}{|c|}{$\begin{array}{l}\text { 95\% Confidence } \\
\text { interval of the } \\
\text { difference }\end{array}$} \\
\hline & & & & & & Lower & Upper \\
\hline IFOF-L & $0.44(0.018)$ & $0.43(0.428)$ & $0.004 *$ & 4 & 0.327 & -0.002 & 0.023 \\
\hline IFOF-R & $0.33(0.012)$ & $0.32(0.012)$ & 0.141 & 4 & 0.162 & -0.001 & 0.015 \\
\hline ILF-L & $0.43(0.012)$ & $0.41(0.021)$ & 0.013 & 4 & 0.276 & 0.006 & 0.027 \\
\hline ILF-R & $0.44(0.014)$ & $0.43(0.023)$ & $0.001 *$ & 4 & 0.387 & 0.006 & 0.027 \\
\hline SFOF-L & $0.34(0.023)$ & $0.32(0.027)$ & 0.107 & 4 & 0.177 & 0.003 & 0.036 \\
\hline SFOF-R & $0.35(0.020)$ & $0.33(0.025)$ & 0.015 & 4 & 0.271 & 0.009 & 0.037 \\
\hline SLF-L & $0.39(0.016)$ & $0.37(0.025)$ & 0.292 & 4 & 0.119 & -0.001 & 0.027 \\
\hline SLF-R & $0.39(0.020)$ & $0.38(0.024)$ & 0.778 & 4 & 0.044 & -0.006 & 0.023 \\
\hline UF-L & $0.28(0.018)$ & $0.27(0.019)$ & 0.009 & 4 & 0.295 & -0.002 & 0.020 \\
\hline UF-R & $0.39(0.014)$ & $0.38(0.015)$ & 0.103 & 4 & 0.179 & -0.001 & 0.019 \\
\hline Callosal body & $0.51(0.010)$ & $0.49(0.020)$ & $0.0005^{*}$ & 4 & 0.403 & 0.010 & 0.029 \\
\hline
\end{tabular}

Table.3-DTI analysis of the FA in white matter tracts in the SC and HC groups. $\mathrm{p}<0.05$ with * for significant p-value. Bonferroni-corrected for multiple comparisons. Age, sex and education were set as covariates.

Abbreviations: HC, healthy control; SC, schizophrenia patients; SD, standard deviation; IFOF-L(-R), left (right) inferior fronto-occipital fasciculus; ILF-L(-R), left (right) inferior long fasciculus; SFOF-L(-R), left (right) superior fronto-occipital fasciculus; SLF-L(-R), left (right) superior long fasciculus; UF-L(-R), left (right) uncinate fasciculus. 
Table.4 Mean value of functional connectivity between regions in $\mathrm{SN}$ and regions in $\mathrm{CEN}$

\begin{tabular}{|c|c|c|c|c|c|c|c|}
\hline \multirow[t]{2}{*}{$\begin{array}{l}\text { Region } \\
\text { (SN) }\end{array}$} & \multirow[t]{2}{*}{$\begin{array}{l}\text { Region } \\
(\mathrm{CEN})\end{array}$} & $\begin{array}{l}\text { HC group } \\
\qquad(\mathrm{n}=21)\end{array}$ & $\begin{array}{l}\text { SC group } \\
\quad(n=22)\end{array}$ & \multirow[t]{2}{*}{$\mathrm{P}$ value } & \multirow[t]{2}{*}{$\begin{array}{c}\text { Degree } \\
\text { of freedom }\end{array}$} & \multirow[t]{2}{*}{$\begin{array}{l}\text { Partial } \\
\text { ETA square }\end{array}$} & \multirow{2}{*}{$\begin{array}{l}\text { 95\% Confidence } \\
\text { interval of the } \\
\text { difference } \\
\text { Lower /Upper }\end{array}$} \\
\hline & & $\operatorname{Mean}(\mathrm{SD})$ & $\operatorname{Mean}(\mathrm{SD})$ & & & & \\
\hline \multirow[t]{4}{*}{$\overline{1 \mathrm{ACC}}$} & IDLPFC & $0.42(0.207)$ & $0.34(0.216)$ & 0.635 & 4 & 0.063 & $-0.079 / 0.201$ \\
\hline & rDLPFC & $0.40(0.230)$ & $0.32(0.236)$ & 0.363 & 4 & 0.105 & $-0.101 / 0.200$ \\
\hline & IPPC & $0.44(0.262)$ & $0.36(0.243)$ & 0.480 & 4 & 0.085 & $-0.085 / 0.244$ \\
\hline & rPPC & $0.44(0.195)$ & $0.34(0.240)$ & 0.350 & 4 & 0.108 & $-0.022 / 0.264$ \\
\hline \multirow[t]{4}{*}{ rACC } & IDLPFC & $0.32(0.177)$ & $0.27(0.194)$ & 0.855 & 4 & 0.034 & $-0.083 / 0.165$ \\
\hline & rDLPFC & $0.44(0.218)$ & $0.41(0.193)$ & 0.415 & 4 & 0.096 & $-0.128 / 0.136$ \\
\hline & IPPC & $0.40(0.259)$ & $0.32(0.217)$ & 0.593 & 4 & 0.069 & $-0.064 / 0.250$ \\
\hline & rPPC & $0.48(0.199)$ & $0.41(0.230)$ & 0.666 & 4 & 0.059 & $-0.062 / 0.223$ \\
\hline \multirow[t]{4}{*}{ IFIC } & IDLPFC & $0.65(0.192)$ & $0.59(0.238)$ & 0.610 & 4 & 0.067 & $-0.093 / 0.191$ \\
\hline & rDLPFC & $0.44(0.247)$ & $0.37(0.206)$ & 0.508 & 4 & 0.081 & $-0.096 / 0.200$ \\
\hline & IPPC & $0.58(0.204)$ & $0.41(0.236)$ & 0.036 & 4 & 0.232 & $0.041 / 0.322$ \\
\hline & rPPC & $0.42(0.201)$ & $0.26(0.258)$ & 0.120 & 4 & 0.171 & $0.022 / 0.323$ \\
\hline \multirow[t]{4}{*}{ rFIC } & IDLPFC & $0.42(0.163)$ & $0.46(0.208)$ & 0.792 & 4 & 0.043 & $-0.175 / 0.073$ \\
\hline & rDLPFC & $0.59(0.228)$ & $0.55(0.225)$ & 0.393 & 4 & 0.100 & $-0.139 / 0.151$ \\
\hline & IPPC & $0.50(0.215)$ & $0.34(0.205)$ & 0.101 & 4 & 0.181 & $0.027 / 0.302$ \\
\hline & rPPC & $0.56(0.196)$ & $0.35(0.239)$ & $0.002 *$ & 4 & 0.354 & $0.075 / 0.339$ \\
\hline
\end{tabular}

Table.4- $\mathrm{fMRI}$ analysis of the functional connectivity between regions in $\mathrm{SN}$ and areas in CEN in the $\mathrm{SC}$ and $\mathrm{HC}$ groups. * significant for $\mathrm{p}<0.05$, Bonferroni-corrected. Age, sex and education were controlled.

Abbreviations: SN, salience network; CEN, central executive network; HC, healthy control; SC, schizophrenia patients; SD, standard deviation. 
Table.5 Relationship between FA in ILF-R and functional connectivity of rFIC-rPPC

\begin{tabular}{|c|c|c|c|c|c|}
\hline Group & $\mathrm{P}$ value & Degree & Effect size & $\begin{array}{l}95 \% \text { Confidence } \\
\text { difference }\end{array}$ & interval of the \\
\hline & & of freedom & r-value & Lower & Upper \\
\hline $\mathrm{HC}$ & 0.213 & 16 & -0.201 & -11.916 & 5.277 \\
\hline $\mathrm{SC}$ & 0.039 & 17 & 0.415 & -0.714 & 12.281 \\
\hline
\end{tabular}

Table.5-Relationship between FA in ILF-R and functional connectivity of rFIC-rPPC in the HC and SC groups. Age, sex and education were controlled as covariates.

Abbreviations: HC, healthy control; SC, schizophrenia patients. 
Caption for FIG.1:

FIG.1 - Regions of significant FA reduction in schizophrenia patients compared with healthy controls. Red-Yellow indicates $\mathrm{p}$ value $(\mathrm{p}<0.05)$. Results are shown overlaid on the Montreal Neurologic Institute $1 \mathrm{~mm}$ template $(Z=70, Z=85, Z=100$ from left to right, respectively) and the mean FA skeleton (green). Threshold-free cluster enhancement method is used. Number of permutation is 5000. $\mathrm{P}<0.05$ for significant $\mathrm{p}$-value and FWE-corrected for multiple comparisons.

\section{Caption for FIG.2:}

Fig.2-Visualization of difference in functional connectivity between $\mathrm{HC}$ and SC on BrainNet Viewer. Brain areas were rendered on the transparent surface of the BrainMesh_ICBM152 template. Red and yellow nodes represent brain regions in SN and CEN, respectively. The pink line stands for functional connectivity which is significantly lower in SC than HC. The grey lines show functional connectivity without significant difference between two groups.

\section{Caption for FIG.3:}

Fig.3-Relationship between FA in ILF-R and functional connectivity of rFIC-rPPC in HC and SC. A significant, positive correlation between the mean FA values in the ILF-R and functional connectivity in rFIC-rPPC is detected in patients with schizophrenia $(r=0.415, p=0.039)$, but not in the healthy control $(\mathrm{p}=0.213)$. Covariates included age, sex and education.

Abbreviations: HC, healthy control; SC, schizophrenia patients; ILF-R, right inferior long fasciculus. 


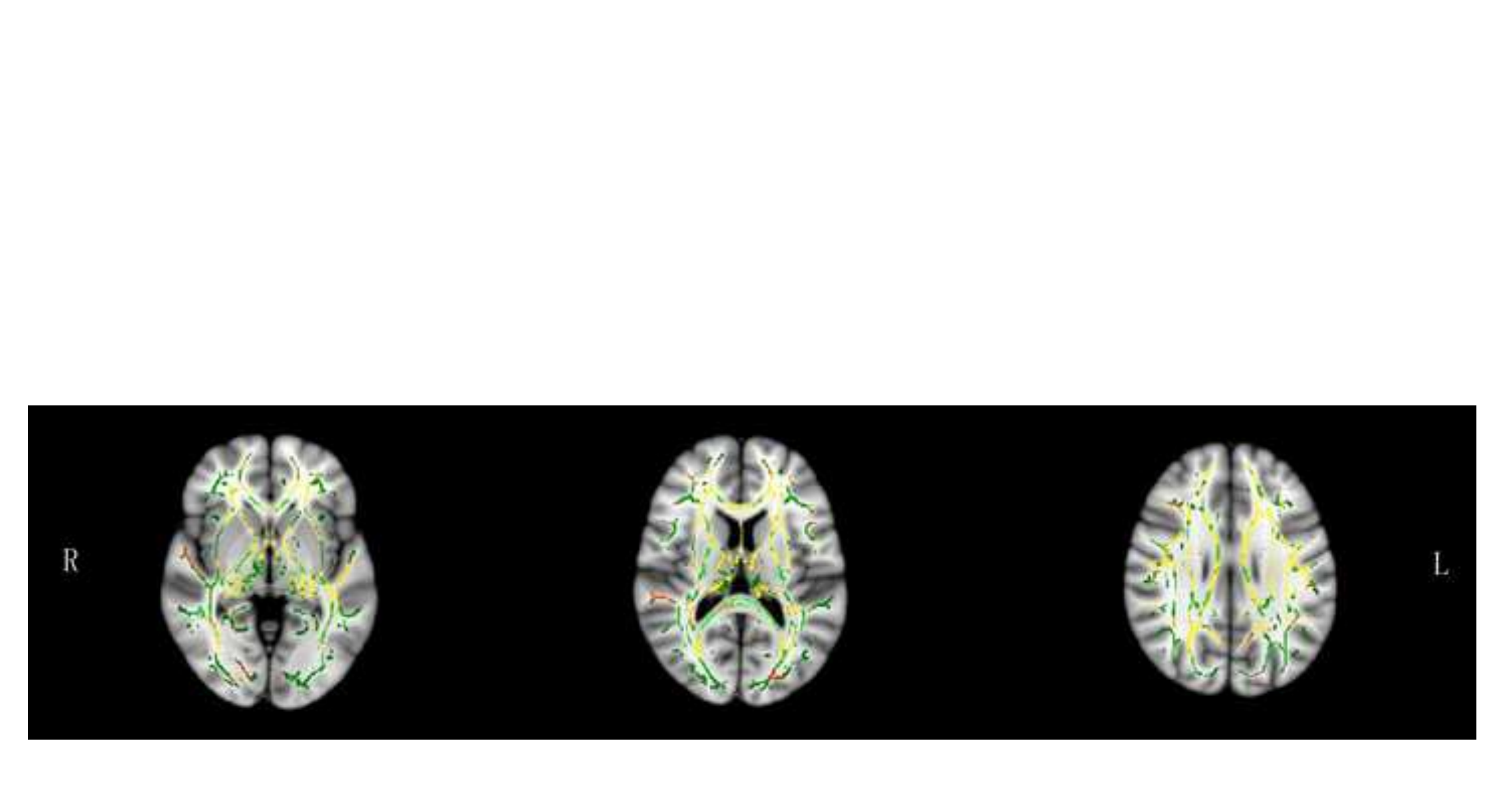

.

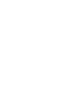

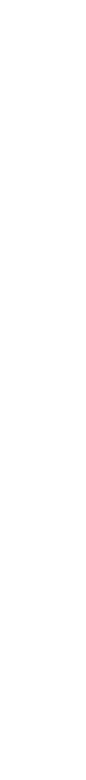

.
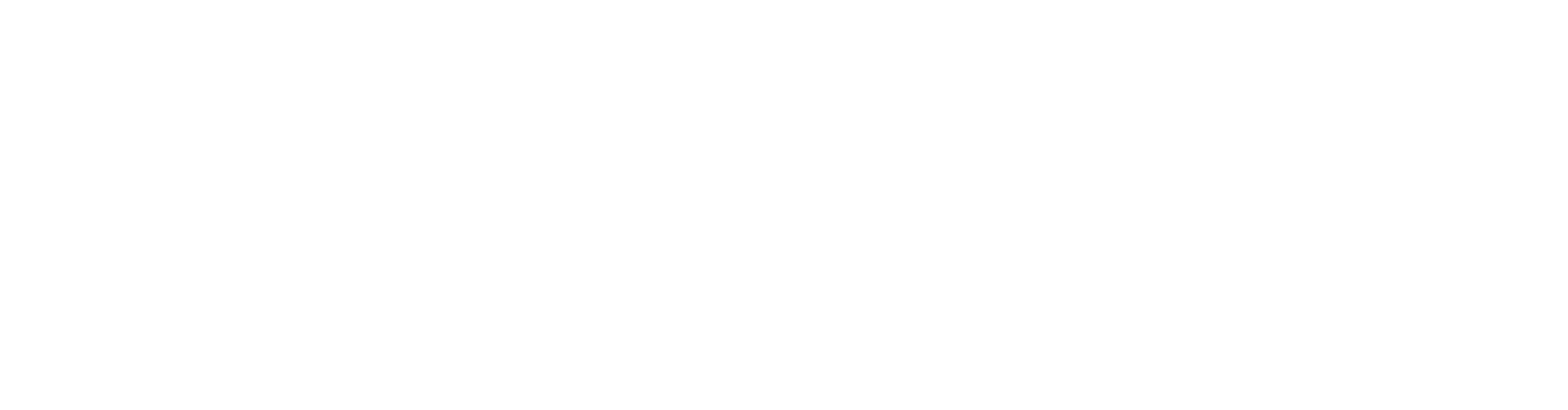

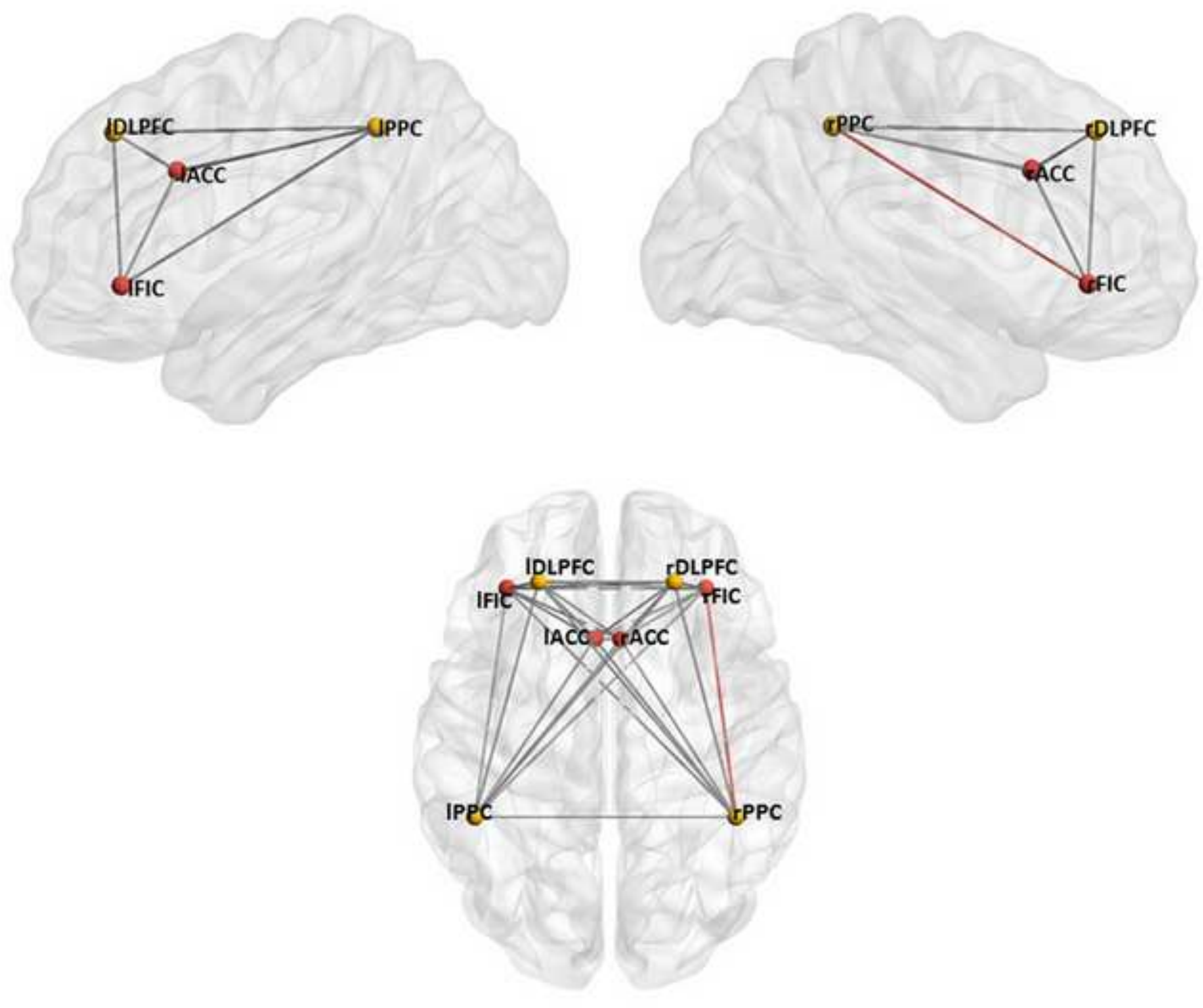
Relationship between FA in II.F.R and functional coninectivin of rFIC-rPPC in HC

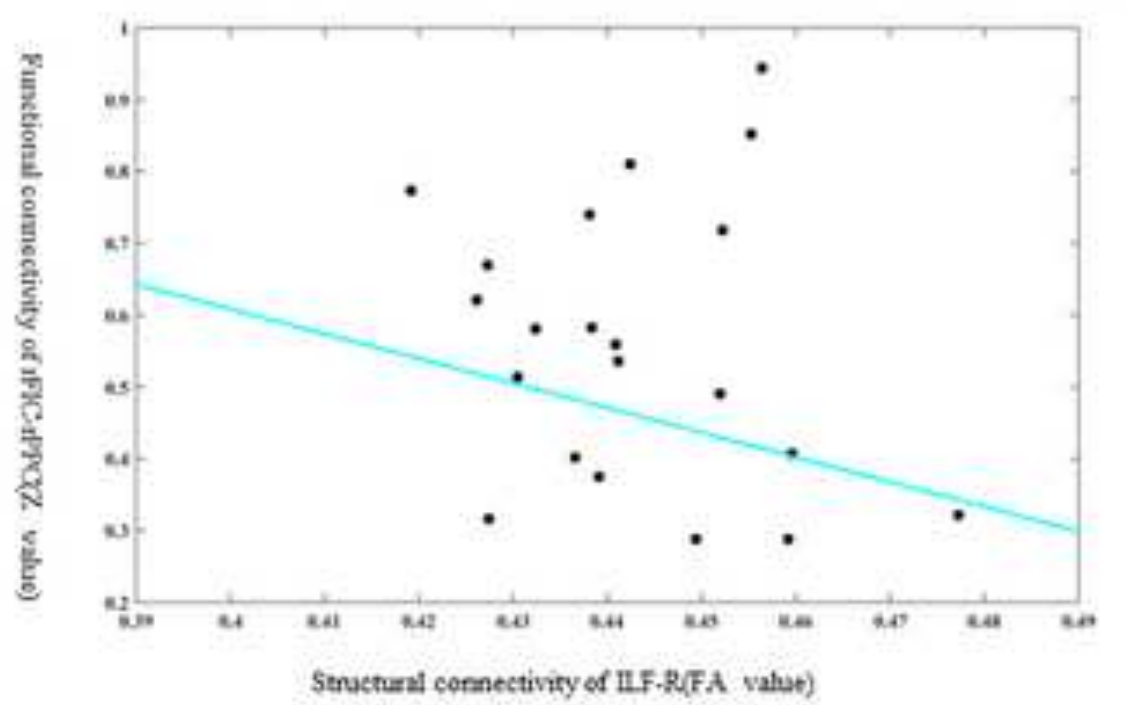

Relationship berneen FA in ILF-R and functional cenectirity of rEIC-rPPC in SC

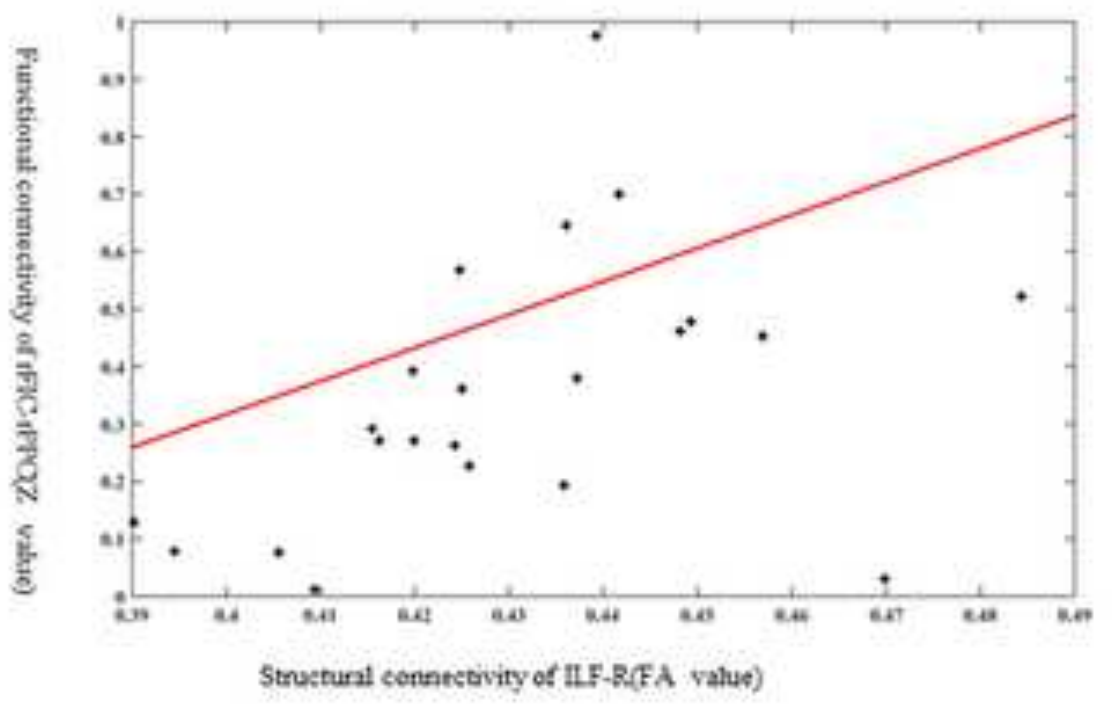

